\title{
A short note on Spector's proof of consistency of analysis
}

\author{
Fernando Ferreira \\ Universidade de Lisboa
}

February 5, 2012

\begin{abstract}
In 1962, Clifford Spector gave a consistency proof of analysis using so-called bar recursors. His paper extends an interpretation of arithmetic given by Kurt Gödel in 1958. Spector's proof relies crucially on the interpretation of the so-called (numerical) double negation shift principle. The argument for the interpretation is ad hoc. On the other hand, William Howard gave in 1968 a very natural interpretation of bar induction by bar recursion. We show directly that, within the framework of Gödel's interpretation, (numerical) double negation shift is a consequence of bar induction.
\end{abstract}

The 1958 paper [4] of Kurt Gödel presented an interpretation (now known as the dialectica interpretation) of Heyting arithmetic HA into a quantifier-free calculus $\mathrm{T}$ of finite-type functionals. The terms of $\mathrm{T}$ denote certain computable functionals of finite type (a primitive notion in Gödel's paper, as it were): the so-called primitive recursive functionals in the sense of Gödel. These terms can be rigorously defined and they include as primitives the combinators (a burocracy of terms for dealing with the "logical" part of the calculus) and the arithmetical constants: 0, the successor constant and, importantly, the recursors. ${ }^{1}$ The dialectica interpretation assigns to each formula $A$ of the language of first-order arithmetic a (quantifier-free) formula $A_{D}(x, y)$ of the language of $\mathrm{T}$, and Gödel showed that if $\mathrm{HA} \vdash A$, then there is a term $t$ (in which $y$ does not occur free) of the language of $\mathrm{T}$ such that $\mathrm{T} \vdash A_{D}(t, y) .{ }^{2}$ The combinators play a central role in showing the preservation of the interpretation under (intuitionistic) logic and, unsurprisingly, the recursors play an essential role in interpreting the induction axioms.

It is convenient to extend the dialectica interpretation to Heyting arithmetic in all finite types $\mathrm{HA}^{\omega}{ }^{3}{ }^{3}$ Within the language of this theory, one can formulate the characteristic principles of the interpretation:

\footnotetext{
${ }^{1}$ The reader can consult [11], [1] or [7] for a precise description of the calculus $\mathrm{T}$ and of its terms in particular. These are good references for details concerning the dialectica interpretation.

${ }^{2}$ We are taking some liberties here (and will take in the sequel). Rigorously, either one should speak of tuple of variables $x:=x_{1}, \ldots, x_{n}$ and $y:=y_{1}, \ldots, y_{m}$ or allow convenient product types.

${ }^{3}$ Some delicate issues concerning equality in higher types arise at this point (if not before). See [1] for a discussion of these matters.
} 


$$
\begin{aligned}
& \mathrm{AC}^{\omega}: \forall x \exists y B(x, y) \rightarrow \exists f \forall x B(x, f(x)), \\
& \mathrm{MP}^{\omega}: \neg \forall x A(x) \rightarrow \exists x \neg A(x), \\
& \mathrm{IP}_{\forall}^{\omega}:(\forall x A(x) \rightarrow \exists y C(y)) \rightarrow \exists y(\forall x A(x) \rightarrow C(y)),
\end{aligned}
$$

where $x$ and $y$ may be of any finite type, $A$ is quantifier-free and $B, C$ are arbitrary. The first principle is a form of choice, the second is a finite-type form of Markov's principle, and the third is an independence of premises statement. These principles arise in virtue of the very definition of the assignment $A \rightsquigarrow A_{D}$ and, accordingly, have trivial interpretations. They are sufficient to prove the equivalence between a given formula $A$ of the language of $\mathrm{HA}^{\omega}$ and its dialectica translation $A^{D}:=\exists x \forall y A_{D}(x, y){ }^{4}$

The dialectica interpretation extends to (finite-type) Peano arithmetic PA ${ }^{\omega}$ by composing it with a negative translation $A \rightsquigarrow A^{g}$. Therefore, if $A$ is a consequence of $\mathrm{PA}^{\omega}$ then $A^{g}$ is provable in $\mathrm{HA}^{\omega}$ and, hence, there is a term $t$ of the language of $\mathrm{T}$ such that $T \vdash\left(A^{g}\right)_{D}(t, y)$.

In the last paragraph of his 1958 paper, Gödel suggests the construction of systems stronger than T. Presumably, calculi with more terms can interpret (via de dialectica blueprint) stronger systems of arithmetic. In this note, we are concerned with the system obtained from $\mathrm{PA}^{\omega}$ by adding full numerical comprehension (obviously, this theory contains full second-order arithmetic, a.k.a. analysis). The formulation of the numerical comprehension principle CA in finite type arithmetic takes the form

$$
\exists f \forall n(f n=0 \leftrightarrow A(n)),
$$

for arbitrary formulae $A$ ( $n$ is a number variable). Clifford Spector isolated in [10] the so-called principle of numerical double negation shift (principle $F$ in Spector's own paper):

$$
\forall n \neg \neg P(n) \rightarrow \neg \neg \forall n P(n),
$$

where $n$ is a number variable and $P$ is arbitrary. This principle is important because of the following result: ${ }^{5}$

Theorem (Kreisel). The negative translation of $\mathrm{CA}$ is provable in the theory $\mathrm{HA}^{\omega}+\mathrm{AC}^{\omega}$ together with the principle of numerical double negation shift.

Proof. The theory HA ${ }^{\omega}$ proves $\forall n \neg \neg\left(A^{g}(n) \vee \neg A^{g}(n)\right)$. By numerical double negation shift, $\neg \neg \forall n\left(A^{g}(n) \vee \neg A^{g}(n)\right)$. Equivalently, $\neg \neg \forall n \exists k\left(\left(k=0 \rightarrow A^{g}(n)\right) \wedge\left(k \neq 0 \rightarrow \neg A^{g}(n)\right)\right)$. Therefore, by $\mathrm{AC}^{\omega}, \neg \neg \exists f \forall n\left(\left(f n=0 \rightarrow A^{g}(n)\right) \wedge\left(f n \neq 0 \rightarrow \neg A^{g}(n)\right)\right)$. This formula is intuitionistically equivalent to the negative translation of $C A$.

\footnotetext{
${ }^{4}$ This result is due to Mariko Yasugi in [14]. There is a brief discussion in [3] clarifying the role of the characteristic principles.

${ }^{5}$ See $[8]$ and note 4 of $[10]$.
} 
Spector's paper [10] was published posthumously. ${ }^{6}$ The paper was submitted by Kreisel on Spector's behalf. The first footnote (written by Kreisel, as were all footnotes) and a postscript by Gödel explain the origin of the paper. In the paper, Spector generalizes Brouwer's principle of bar induction to higher types. ${ }^{7}$ We work with the following version of bar induction: For any given formulas $A$ and $B$, if

(1) $\forall f \exists k A(\bar{f}(k))$

(2) $\forall s, s^{\prime}\left(A\left(s^{\prime}\right) \wedge s^{\prime}\right.$ is a initial subsequence of $\left.s \rightarrow A(s)\right)$

(3) $\forall s(A(s) \rightarrow B(s))$

(4) $\forall s(\forall x B(\hat{s x}) \rightarrow B(s))$

then $B(\langle\rangle)$. In the above, $x$ is of an arbitrary given type, $f$ is an infinite sequence of elements of the type of $x$, and $s, s^{\prime}$ are finite sequences of elements of the type of $x$ (also, $\bar{f}(k)$ is the finite sequence $\langle f 0, \ldots, f(k-1)\rangle$ and $\hat{s x}$ is the sequence $s$ concatenated with the element $x){ }^{8,9}$ The great novelty is that Spector also introduces definitions by so-called bar recursion. ${ }^{10}$ There are now new terms, arising from the bar recursors: an extension of Gödel's T calculus has been produced. Spector points that "since our immediate objective is to obtain a quantifier-free interpretation of analysis, bar recursion rather than bar induction is appropriate." The goal is, of course, to witness (with the help of the new terms) the dialectica translations of the instances of the numerical double negation shift principle. By Kreisel's result above, this would solve the problem of providing an interpretation for CA. In the crucial Section 10 of his paper, Spector produces an ad hoc witness solution. ${ }^{11}$

\footnotetext{
${ }^{6}$ Spector died at the age of thirty in the summer of 1961 of acute leukemia, after spending the academic year in the Institute for Advanced Study in Princeton, New Jersey.

${ }^{7}$ A statement of bar induction (or bar theorem) by Brouwer himself can be found in [2]. Van Atten has a discussion of this principle in his book [12] on Brouwer.

${ }^{8}$ The usual presentation of finite type arithmetic has no primitive types for finite sequences (of a given fixed type). However, there are manners of representing finite sequences in finite type arithmetic. We will not worry about these issues here.

${ }^{9}$ Bar induction in the sense of Brouwer demands that $x$ be a number variable. Brouwerian bar induction is already sufficient to interpret $\Sigma_{1}^{0}$ comprehension (and so, by a well-known fact, arithmetical comprehension). This can be seen by analyzing carefully the proofs of the Kreisel result above and of the main result below. Spector introduced bar induction for $x$ of any given type.

${ }^{10} \mathrm{We}$ do not define bar recursion in this paper (for modern references and discussions, see note 1). In contrast to recursion, bar recursion is not well defined in the full set theoretic structure of finite type functionals (it is not a classical principle). It is nevertheless defined in the structure of continuous functionals and in the strong majorizability model. Bar induction is, on the other hand, a classical principle.

${ }^{11}$ The heart of the matter is the solution of a system of equations arising from the dialectica translation of the principle of double negation shift. Due the presence of many negations, this system is rather cryptic. It is a kind of brute fact that, somehow, one is able to solve it with bar recursive functionals. (This is not to say that the proof in unmotivated, as Spector describes in his paper the motivation for his proof.) The intuitionistic laws $\neg \neg A_{1} \wedge \ldots \wedge \neg \neg A_{n} \rightarrow \neg \neg\left(A_{1} \wedge \ldots \wedge A_{n}\right)$ are miniaturizations of the principle of
} 
Why does Spector introduce and discuss bar induction? After all, his interpretation of analysis does not formally require it. He explains that "bar induction (is discussed) primarily to point out the relationship between bar recursion and the bar theorem" and candidly observes that "bar recursion is a principle of definition and bar induction a corresponding principle of proof." This is exactly right. The situation is analogous with that of induction/recursion (pace the remark in note 10). In effect, William Howard proved in [6] that the principle of bar induction is interpretable in the extended calculus, with bar recursors. The proof is very natural and, if I may add, has a certain character of inexorability about it. $^{12}$

So, there is this very satisfying picture:

\section{induction bar induction}

recursion

bar recursion

The following result shows that the principle of double negation shift follows from bar induction and, therefore (by Howard), has a dialectica interpretation in the extended calculus.

Theorem. In the theory $\mathrm{HA}^{\omega}$ together with the characteristic principles, the principle of bar induction implies numerical double negation shift.

Proof. Under the hypothesis of the theorem, we must show that $\forall n \neg \neg P(n) \rightarrow \neg \neg \forall n P(n)$ is a consequence of bar induction ( $P$ arbitrary). By the characteristic principles, the formula $P(n)$ is equivalent to a formula of the form $\exists x \forall y Q(x, y, n)$, with $Q$ quantifierfree. Let us assume $\forall n \neg \neg P(n)$ and $\neg \forall n P(n)$ in order to derive a contradiction. Consider $A(s): \equiv \exists i<|s| \exists y \neg Q\left(s_{i}, y, i\right)$ and $B(s): \equiv A(s)$, where $s$ is of the type of finite sequences of elements of type $x(|s|$ denotes the length of $s)$. It is clear that $\neg B(\langle\rangle)$. Therefore, if one proves the hypothesis (1) to (4) of the principle of bar induction, we get a contradiction. Hypothesis (2) and (3) hold trivially. Hypothesis (1) is a consequence of the assumption that $\neg \forall n P(n)$. In effect, this assumption says that $\neg \forall n \exists x \forall y Q(x, y, n)$. By $\mathrm{AC}^{\omega}$ and intuitionistic logic, we get $\forall f \neg \forall n, y Q(f n, y, n)$. Markov's principle MP ${ }^{\omega}$, on the other hand, entails $\forall f \exists n, y \neg Q(f n, y, n)$. Therefore, $\forall f \exists k \exists i<k \exists y \neg Q(f i, y, i)$, i.e., $\forall f \exists k A(\bar{f}(k))$.

Let us argue (4). Given a finite sequence $s$, suppose $\forall x B(\hat{s x})$, i.e.:

$$
\forall x\left(\exists i<|s| \exists y \neg Q\left(s_{i}, y, i\right) \vee \exists y \neg Q(x, y,|s|)\right) .
$$

double negation shift and, by Gödel's paper, their dialectica translations have witnessing solutions in the T calculus. It is a non trivial exercise to find such solutions, even for $n=2$. Paulo Oliva in [9] discusses these solutions in detail and tries to motivate the use of bar recursion for the dialectica interpretation of double negation shift in terms of a limit process when the number of conjuncts goes to infinity.

${ }^{12}$ Avigad and Feferman say in [1] that "while the proof requires some effort, the underlying idea is straightforward." 
By the assumption $\forall n \neg \neg P(n)$, we have $\neg \neg P(|s|)$. Using intuitionistic logic and Markov's principle $\mathrm{MP}^{\omega}$, we get $\neg \forall x \exists y \neg Q(x, y,|s|)$. We now appeal to the intuitionistic law

$$
\forall x(\phi \vee \psi(x)), \neg \forall x \psi(x) \Rightarrow \neg \neg \phi
$$

(where $x$ does not occur free in $\phi$ ) to infer $\neg \neg \exists i<|s| \exists y \neg Q\left(s_{i}, y, i\right)$. By Markov's principle $\mathrm{MP}^{\omega}$ once again, we finally conclude $B(s)$.

Spector's paper has an appendix in which he aims to "indicate how bar induction can be used to obtain an interpretation (of analysis) in a system with quantifiers" (cf. p. 8 of [10]). I read this statement as proposing to show that the translations $A^{D}$ of instances $A$ of (negative translations of) numerical comprehension somehow follow from bar induction. Rather than presenting an $x$-witnessing term for $A_{D}(x, y)$, Spector sets himself the goal of proving the statement $\exists x \forall y A_{D}(x, y)$. Section 12.1 of the appendix describes an informal proof of this result for the particular case of comprehension for $\Sigma_{1}^{0}$ predicates. $^{13}$ Our result above can be viewed as providing a formal argument for the general case. ${ }^{14,15}$

\section{References}

[1] J. Avigad and S. Feferman. Gödel's functional ("Dialectica") interpretation. In S. R. Buss, editor, Handbook of Proof Theory, volume 137 of Studies in Logic and the Foundations of Mathematics, pages 337-405. North Holland, Amsterdam, 1998.

[2] L. E. J. Brouwer. Über Definitionsbereiche von funktionen. Mathematische Annalen, 93:60-75, 1927. English translation in [13], pp. 457-463.

[3] F. Ferreira. A most artistic package of a jumble of ideas. dialectica, 62:205-222, 2008. Special Issue: Gödel's dialectica Interpretation. Guest editor: Thomas Strahm.

[4] K. Gödel. Über eine bisher noch nicht benützte Erweiterung des finiten Standpunktes. dialectica, 12:280-287, 1958. Reprinted with an English translation in [5], pp. 240-251.

[5] K. Gödel. Collected Works, Vol. II. S. Feferman et al., eds. Oxford University Press, Oxford, 1990.

[6] W. A. Howard. Functional interpretation of bar induction by bar recursion. Compositio Mathematica, 20:107-124, 1968.

\footnotetext{
${ }^{13}$ It is Spector himself that says that the proof is informal. It uses a Brouwerian continuity principle and it is best seen as an argument in Brouwerian (intuitionistic) analysis. Note that the proof only uses bar induction of numerical type (cf. note 9).

${ }^{14}$ According to a letter of Spector to Kreisel mentioned in the first footnote of [10], this seemed to be the intent of Spector were he able to complete his paper.

${ }^{15}$ The main theorem of this note can also be obtained from the work of Howard in [6]. Our proof has the advantage of being very direct.
} 
[7] U. Kohlenbach. Applied Proof Theory: Proof Interpretations and their Use in Mathematics. Springer Monographs in Mathematics. Springer, Berlin, 2008.

[8] G. Kreisel. Interpretation of analysis by means of constructive functionals of finite types. In A. Heyting, editor, Constructivity in Mathematics, pages 101-128. North Holland, Amsterdam, 1959.

[9] P. Oliva. Understanding and using Spector's bar recursive interpretation of classical analysis. In B. Löwe A. Beckmann, U. Berger and J. Tucker, editors, Logical Approaches to Computational Barriers - Proceedings of Computability in Europe 2006, volume 3988 of Lecture Notes in Computer Science, pages 423-434. Springer, New York, 2006.

[10] C. Spector. Provably recursive functionals of analysis: a consistency proof of analysis by an extension of principles in current intuitionistic mathematics. In F. D. E. Dekker, editor, Recursive Function Theory: Proceedings of Symposia in Pure Mathematics, volume 5, pages 1-27. American Mathematical Society, Providence, Rhode Island, 1962 .

[11] A. S. Troelstra (ed.). Metamathematical Investigation of Intuitionistic Arithmetic and Analysis, volume 344 of Lecture Notes in Mathematics. Springer, Berlin, 1973.

[12] M. van Atten. On Brouwer. Wadsworth, 2004.

[13] J. van Heijenoort (ed.). From Frege to Gödel. Harvard University Press, 1967.

[14] M. Yasugi. Intuitionistic analysis and Gödel's interpretation. Journal of the Mathematical Society of Japan, 15:101-112, 1963. 